\title{
Padrão de sono em crianças de 12 a 36 meses de idade
}

\author{
Sleep pattern in children aged 12 to 36 months age \\ Patrón del sueño de niños de 12 a 36 meses de edad
}

\begin{abstract}
Catarina Andreia Rosa Saraiva Marinho $0^{1,2}$ (D) João Carvalho Duarte ${ }^{3}$ (D) Cândida Koch ${ }^{4}$ (D) Margarida Reis Santos ${ }^{1,4}$ (D)
\end{abstract}

1. Universidade do Porto, Instituto de Ciências Biomédicas Abel Salazar. Porto, Portugal.

2. Centro Hospitalar Tondela-Viseu. Viseu, Portugal.

3. Instituto Politécnico de Viseu, Escola Superior de Saúde de Viseu. Viseu, Portugal.

4. Escola Superior de Enfermagem do Porto. Porto, Portugal.
Autor correspondente:

Catarina Andreia Rosa Saraiva Marinho. E-mail: catarin_marinho@hotmail.com

Recebido em 22/07/2021.

Aprovado em 27/10/2021.

DOI:https://doi.org/10.1590/2177-9465-EAN-2021-0269

\section{Resumo}

Objetivo: Avaliar o padrão de sono de crianças de 12 a 36 meses. Método: Estudo transversal, realizado em uma amostra de 808 crianças com 12-36 meses de idade, que frequentavam creches no distrito de Viseu, Portugal. Coleta de dados realizada por meio de questionário aplicado aos pais, entre novembro de 2018 e setembro de 2019. Estudo aprovado pela comissão de ética do Instituto de Ciências Biomédicas Abel Salazar, Portugal. Resultados: Verificou-se que durante a semana as crianças dormiam, por dia, entre $9 \mathrm{~h} 30 \mathrm{~m}$ e $18 \mathrm{~h}(\mathrm{M}=12 \mathrm{~h} 25 \mathrm{~m} \pm 1 \mathrm{~h} 02 \mathrm{~m})$ e no fim de semana, entre $9 \mathrm{~h}$ e $19 \mathrm{~h}(\mathrm{M}=12 \mathrm{~h} 49 \mathrm{~m} \pm 1 \mathrm{~h} 15 \mathrm{~m})$. No fim de semana, as crianças deitavam-se e acordavam mais tarde que à semana $(M=21 \mathrm{~h} 42 \mathrm{~m} \pm 40 \mathrm{~m}, \mathrm{M}=8 \mathrm{~h} 15 \mathrm{~m} \pm 50 \mathrm{~m}$, respetivamente) A maioria não dormia a sesta da manhã, somente a da tarde. Conclusão: Os resultados indicam que a maioria das crianças (91,5\% semana; $85,6 \%$ fim de semana) dorme o número de horas recomendado. As mais novas são as que dormem mais Implicações para a prática: É importante que, nas consultas de vigilância de saúde, os enfermeiros realizem educação para a saúde direcionada aos pais sobre a relevância de manter um padrão de sono adequado à criança.

Palavras chave: Enfermagem; Lactente; Pré-Escolar; Saúde da criança; Sono.

\section{Abstract}

Objective: To evaluate the sleep pattern in children aged 12 to 36 months. Method: Cross-sectional study, carried out in a sample of 808 children aged 12-36 months who attended day care centers in the district of Viseu, Portugal. The data was collected through a questionnaire applied to parents between November 2018 and September 2019. The study was approved by the Ethics Committee of Instituto de Ciências Biomédicas Abel Salazar, Portugal. Results: The children were found to sleep between $9 \mathrm{~h}$ $30 \mathrm{~min}$ and $18 \mathrm{~h}(\mathrm{M}=12 \mathrm{~h} 25 \mathrm{~min} \pm 1 \mathrm{~h} 02 \mathrm{~min})$ per day during the week and between $9 \mathrm{~h}$ and $19 \mathrm{~h}(\mathrm{M}=12 \mathrm{~h} 49 \mathrm{~min} \pm 1 \mathrm{~h} 15 \mathrm{~min})$ on weekends. The children went to bed and woke up later on weekends than during the week ( $M=21 \mathrm{~h} 42 \mathrm{~min} \pm 40 \mathrm{~min}, \mathrm{M}=8 \mathrm{~h}$ $15 \mathrm{~min} \pm 50 \mathrm{~min}$, respectively). Most did not take morning naps, but only afternoon naps. Conclusion: The results indicate that most children ( $91.5 \%$ on weekdays; $85.6 \%$ on the weekend) sleep the recommended number of hours. The youngest are the ones who sleep the most. Implications for practice: It is important that, during child health surveillance consultations, nurses provide health education to parents about the importance of maintaining a sleep pattern that is adequate for their child's age.

Keywords: Nursing; Infant; Preschool; Child health; Sleep.

\section{Resumen}

Objetivo: Evaluar el patrón de sueño de niños de 12 a 36 meses. Método: Estudio transversal, realizado en una muestra de 808 niños de 12 a 36 meses que asistían a guarderías en el distrito de Viseu, Portugal. La recolección de datos se realizó mediante cuestionario aplicado a padres entre noviembre de 2018 y septiembre de 2019. El estudio fue aprobado por el Comité de Ética del Instituto de Ciências Biomédicas Abel Salazar, Portugal. Resultados: Se encontró que en los días laborables los niños dormían, por día, entre 9h 30m y $18 \mathrm{~h}(\mathrm{M}=12 \mathrm{~h} 25 \mathrm{~m} \pm 1 \mathrm{~h} 02 \mathrm{~m})$ y los fines de semana entre $9 \mathrm{~h}$ y $19 \mathrm{~h}(\mathrm{M}=12 \mathrm{~h} 49 \mathrm{~m} \pm 1 \mathrm{~h} 15 \mathrm{~m})$. Los fines de semana los niños se acostaban y se despertaban más tarde que en la semana $(M=21 \mathrm{~h} 42 \mathrm{~m} \pm 40 \mathrm{~m}, M=8 \mathrm{~h} 15 \mathrm{~m} \pm 50 \mathrm{~m}$, respectivamente). La mayoría no dormía la siesta por la mañana, pero sí por la tarde. Conclusión: Los resultados indican que la mayoría de los niños (91,5\% en días laborables, 85,6\% en fines de semana) duermen el número de horas recomendado. Los más pequeños son los que más duermen. Implicaciones para la práctica: Es importante que, en las consultas de vigilancia de la salud infantil, las enfermeras brinden educación sanitaria a los padres sobre la importancia de mantener un patrón de sueño adecuado a la edad del niño.

Palabras clave: Enfermería; Infantil; Preescolar; Salud de los niños; Sueño. 


\section{INTRODUÇÃO}

O sono é um parâmetro importante para o desenvolvimento infantil e a sua relevância tem sido associada a um desenvolvimento físico, cognitivo e comportamental saudável. Tem-se mostrado tão importante para a sobrevivência humana quanto a alimentação. A maioria das crianças pequenas (entre os 12 e os 36 meses de idade) passa metade ou mais de metade do dia dormindo ${ }^{1}$.

O sono é uma função natural, básica e sujeita a uma evolução contínua ao longo da vida. Qualquer ser humano passa, pelo menos, um terço da vida dormindo².

Como processo fisiológico, o sono está estruturado em sono REM (movimento rápido de olhos - sono mais leve) e sono NREM (movimento de olhos não rápido - sono mais profundo) ${ }^{3}$. A atividade neuronal varia com o estado de sono, sendo menor do que em vigília durante o sono NREM e maior do que em vigília no período REM ${ }^{4}$.

Sabe-se que o padrão de sono se apresenta com um ritmo circadiano a cada 24 horas e que o sono não é um processo contínuo, processando-se em ciclos que mudam durante os primeiros anos de vida ${ }^{4}$, havendo alterações significativas e variabilidade individual no padrão de sono ao longo do desenvolvimento ${ }^{5}$. O ciclo diário de sono-vigília depende da interação de dois fatores: do processo homeostático do sono e do processo circadiano, designado como o "Modelo de Duas Partes". No ciclo de sono, aos 11 meses de idade, o sono REM dura 10-45 minutos, NREM cerca de 20 minutos e a transição perto de 10 minutos $^{4}$.

É impossível um indivíduo sobreviver sem dormir e, para o desenvolvimento físico, mental e intelectual saudáveis, é imprescindível o seu cumprimento regular.

Existem vários fatores que podem desencadear problemas de sono. Algumas vezes a causa é desconhecida. Outras vezes, está associada a condições médicas (insuficiência cardíaca, síndrome das pernas inquietas, cefaleias), a condições psicológicas (depressão, ansiedade) ou a outros fatores (problemas ambientais, uso de medicamentos, trauma infantil) ${ }^{3}$.

Em lactentes e pré-escolares, a importância do sono relaciona-se com fatores do desenvolvimento da criança nas áreas da linguagem, memória e comportamento ${ }^{6-8}$.

Diversas entidades, nacionais e internacionais, estimam que crianças entre os 12 e 36 meses de idade deveriam dormir entre 11 e 14 horas por dia e que, idealmente, entre os 12 e 24 meses, ter 10 a 11 horas de sono noturno e 2 a 4 horas em sestas, enquanto aos 36 meses deveriam dormir entre 10 e 11 horas por noite e 1 a 3 horas em sestas ${ }^{9-11}$.

Considerando a idade das crianças, a família desempenhará um papel fundamental na aquisição de hábitos de sono saudáveis. Sabese que o sono nem sempre é um tema abordado adequadamente nas consultas de Enfermagem ${ }^{1}$. Nesse contexto, os profissionais de saúde, em especial os enfermeiros, também têm por missão alertar para a importância dessa atividade de vida, visando o desenvolvimento saudável da criança e proporcionando um suporte à família para a consciencialização sobre as possíveis consequências. Com este estudo, objetiva-se avaliar o padrão do sono de crianças entre os 12 e 36 meses que frequentam creches no distrito de Viseu, Portugal, durante a semana e fim de semana, avaliando a distribuição das horas de sono noturno e das sestas durante esses períodos.

\section{MÉTODO}

Estudo exploratório, transversal, descritivo e relacional. A população alvo foram crianças entre os 12 e 36 meses de idade que frequentavam creches no distrito de Viseu, Portugal, configurando uma amostra não probabilística por conveniência. Contactaram-se as 94 instituições particulares de solidariedade social (IPSS) e creches privadas do distrito de Viseu. Das 68 $(72,3 \%)$ que aceitaram participar do estudo, 62 eram IPSS e 6 eram creches privadas.

Após um pré-teste do instrumento numa amostra de crianças da mesma faixa etária, a coleta dos dados efetuou-se por questionário entre novembro de 2018 e setembro de 2019.

Os questionários foram entregues aos pais de todas as crianças que frequentavam as instituições que aceitaram integrar o estudo. No momento de entrega, explicaram-se-Ihes a finalidade e os objetivos do estudo. Dos 2036 questionários entregues, foram devolvidos 828 e validados 808 , dado que 20 apresentaram preenchimento incompleto.

O questionário incluía questões visando a caracterização sociodemográfica da criança e o seu padrão de sono. Os dados referentes às horas de sono foram obtidos por meio de questões abertas: hora de deitar, de acordar, duração das sestas da manhã e da tarde, na rotina da semana e no fim de semana. Posteriormente, os dados foram recodificados em três grupos que tiveram em conta as recomendações nacionais e internacionais relativas às horas de sono para crianças dessa faixa etária. Para a análise estatística dos dados, recorreu-se ao IBM Statistical Package for the Social Sciences (SPSS) 25. Os dados foram explorados por meio de estatística descritiva: frequências absolutas e percentuais, medidas de tendência central, nomeadamente média e medidas de dispersão, como a amplitude de variação, o coeficiente de variação e o desvio padrão. Recorreu-se também à estatística inferencial, nomeadamente análise univariada e bivariada. Para toda a análise, a significância estatística foi aceite para valores $p<0,05$ e residuais ajustados $\geq 1,96$.

O estudo foi aprovado pela Comissão de Ética (CETI) do Instituto de Ciências Biomédicas Abel Salazar, Universidade do Porto (parecer n.ำ263/2018/CETI) e autorizado pelos responsáveis das creches implicadas. Todos os participantes assinaram um termo de consentimento informado.

\section{RESULTADOS}

A amostra incluía 808 crianças, das quais $46,5 \%$ tinham idade compreendida entre 12 e 23 meses; 44,1\%, entre 24 e 35 meses; e 9,4\% tinham 36 meses. A maioria (50,4\%) era do sexo masculino.

Relativamente ao número de horas de sono por noite durante a semana, verificou-se uma variação entre $8 \mathrm{~h}$ e $13 \mathrm{~h}$ $(M=10 \mathrm{~h} 22 \mathrm{~m} \pm 42 \mathrm{~m})$ e no fim de semana entre $8 \mathrm{~h} 30 \mathrm{~m}$ e $13 \mathrm{~h}$ $30 \mathrm{~m}(\mathrm{M}=10 \mathrm{~h} 36 \mathrm{~m} \pm 49 \mathrm{~m})$.

\section{Hora de deitar e de acordar}

Verificou-se que durante a semana, $42,8 \%$ das crianças deitavam-se até às $21 \mathrm{~h}$ e $26,7 \%$ deitavam-se depois das $21 \mathrm{~h}$ $30 \mathrm{~m}$ (38,0\% com 12-23 meses, 50,0\% com 24-35 meses, $12,0 \%$ 
Tabela 1. Hora de deitar e de acordar durante a semana e no fim de semana em função da idade da criança. Viseu, Portugal, 2019.

\begin{tabular}{|c|c|c|c|c|c|c|c|c|c|c|c|c|}
\hline \multirow{2}{*}{$\begin{array}{c}\text { Idade } \\
\text { Variáveis }\end{array}$} & \multicolumn{2}{|c|}{$12-23$ meses } & \multicolumn{2}{|c|}{ 24-35 meses } & \multicolumn{2}{|c|}{36 meses } & \multicolumn{2}{|c|}{ Total } & \multicolumn{3}{|c|}{ Residuais } & \multirow[b]{2}{*}{$X^{2}(p)$} \\
\hline & $\begin{array}{c}n \\
(376)\end{array}$ & $-46,50 \%$ & $\begin{array}{c}n \\
(356)\end{array}$ & $-44,10 \%$ & $\begin{array}{c}n \\
(76) \\
\end{array}$ & $-9,40 \%$ & $\begin{array}{c}n \\
(808)\end{array}$ & $-100,00 \%$ & 1 & 2 & 3 & \\
\hline \multicolumn{13}{|c|}{ Hora deitar - Semana } \\
\hline$\leq 21 h$ & 182 & 52,6 & 136 & 39,3 & 28 & 8,1 & 346 & 42,8 & 3 & $-2,4$ & $-1,1$ & \\
\hline$>21 \mathrm{~h} \leq 21 \mathrm{~h} 30 \mathrm{~m}$ & 112 & 45,5 & 112 & 45,5 & 22 & 8,9 & 246 & 30,4 & $-0,4$ & 0,6 & $-0,3$ & $12,1(*)$ \\
\hline$>21 \mathrm{~h} \mathrm{30m}$ & 82 & 38 & 108 & 50 & 26 & 12 & 216 & 26,7 & -3 & 2,1 & 1,5 & \\
\hline \multicolumn{13}{|c|}{ Hora deitar - Fim de semana } \\
\hline$\leq 21 h$ & 134 & 61,5 & 75 & 34,4 & 9 & 4,1 & 218 & 27 & 5,2 & $-3,4$ & $-3,1$ & \\
\hline$>21 \mathrm{~h} \leq 21 \mathrm{~h} 30 \mathrm{~m}$ & 76 & 47,8 & 72 & 45,3 & 11 & 6,9 & 159 & 19,7 & 0,4 & 0,3 & $-1,2$ & $36,4(* * *)$ \\
\hline$>21 \mathrm{~h} 30 \mathrm{~m}$ & 166 & 38,5 & 209 & 48,5 & 56 & 13 & 431 & 53,3 & $-4,9$ & 2,7 & 3,7 & \\
\hline \multicolumn{13}{|c|}{ Hora acordar - Semana } \\
\hline$\leq 7 \mathrm{~h} 30 \mathrm{~m}$ & 162 & 46,3 & 160 & 45,7 & 28 & 8 & 350 & 43,3 & $-0,1$ & 0,8 & $-1,2$ & \\
\hline$>7 \mathrm{~h} 30 \mathrm{~m} \leq 8 \mathrm{~h}$ & 161 & 46,5 & 148 & 42,8 & 37 & 10,7 & 346 & 42,8 & 0 & $-0,6$ & 1,1 & 1,8 \\
\hline$>8 h$ & 53 & 47,3 & 48 & 42,9 & 11 & 9,8 & 112 & 13,9 & 0,2 & $-0,3$ & 0,2 & \\
\hline \multicolumn{13}{|c|}{ Hora acordar - Fim de semana } \\
\hline$\leq 7 \mathrm{~h} 30 \mathrm{~m}$ & 83 & 54,2 & 65 & 42,5 & 5 & 3,3 & 153 & 18,9 & 2,1 & $-0,4$ & $-2,9$ & \\
\hline$>7 \mathrm{~h} 30 \mathrm{~m} \leq 8 \mathrm{~h}$ & 137 & 46 & 136 & 45,6 & 25 & 8,4 & 298 & 36,9 & $-0,2$ & 0,7 & $-0,8$ & $13,9(* *)$ \\
\hline$>8 \mathrm{~h}$ & 156 & 43,7 & 155 & 43,4 & 46 & 12,9 & 357 & 44,2 & $-1,4$ & $-0,3$ & 3 & \\
\hline
\end{tabular}

Abreviaturas: $X^{2}$ - teste de Qui quadrado; ${ }^{* * *} p<0,001,{ }^{* *} p<0,01,{ }^{*} p<0,05$; Fonte: Dados de pesquisa

com 36 meses). Observaram-se diferenças estatisticamente significativas $(p=0,017)$, verificando-se a tendência de as crianças mais novas se deitarem mais cedo $(\leq 21 \mathrm{~h})$, enquanto as crianças com idade entre 24 e 35 meses se deitavam mais tarde (> 21h 30m).

Durante o fim de semana, observou-se que a maioria das crianças $(53,3 \%)$ deitava-se depois das $21 \mathrm{~h} 30 \mathrm{~m} \mathrm{(38,5 \%} \mathrm{com}$ 12-23 meses, 48,5\% com 24-35 meses, 13,0\% com 36 meses) e $27,0 \%$ deitavam-se até às $21 \mathrm{~h}$. As diferenças estatisticamente significativas $(p=0,000)$ sugerem que as crianças mais novas (12-23 meses) tendem a deitar-se mais cedo $(\leq 21 \mathrm{~h})$ do que as mais velhas (24-35 meses e 36 meses).

Constatou-se uma prevalência de $43,3 \%$ de crianças que acordavam até às $7 \mathrm{~h} 30 \mathrm{~m}$ durante a semana. Observaram-se diferenças estatisticamente significativas em relação à hora de acordar ao fim de semana $(p=0,008)$, observando-se a tendência de as crianças mais novas (12-23 meses) acordarem mais cedo ( $\leq 7 \mathrm{~h} 30 \mathrm{~m}$ ) do que as mais velhas (36 meses) (Tabela 1 ).

Verificou-se que a idade tem associação com a hora de deitar e acordar das crianças; as mais velhas se deitavam e acordavam mais tarde, existindo diferenças estatisticamente significativas em relação à hora de deitar durante a semana $(p=0,003)$ e durante o fim de semana $(p=0,000) e$, ainda, em relação à hora de acordar durante o fim de semana ( $p=0,002)$ (Tabela 2$)$.

Relativamente à hora de acordar durante a semana, apurouse que eram as crianças com idade entre 24 e 35 meses as que acordavam mais cedo $(\mathrm{OM}=397,97)$ e as de 36 meses, as que
Tabela 2. Teste de Kruskal-Wallis entre a hora de deitar e de acordar durante a semana e o fim de semana e a idade da criança. Viseu, Portugal, 2019.

\begin{tabular}{|c|c|c|}
\hline Idade da criança & $\begin{array}{c}\text { Ordenação } \\
\text { Média }\end{array}$ & $\mathrm{KW}(\mathrm{p})$ \\
\hline \multicolumn{3}{|l|}{ Hora deitar - Semana } \\
\hline $12-23$ meses & 376,6 & \\
\hline 24-35 meses & 426,5 & $11,7(* *)$ \\
\hline 36 meses & 439,5 & \\
\hline \multicolumn{3}{|c|}{ Hora deitar - Fim de semana } \\
\hline 12-23 meses & 361,1 & \\
\hline 24-35 meses & 431,5 & $34,9(* * *)$ \\
\hline 36 meses & 493,1 & \\
\hline \multicolumn{3}{|l|}{ Hora acordar - Semana } \\
\hline 12-23 meses & 405,8 & \\
\hline 24-35 meses & 397,9 & 1,3 \\
\hline 36 meses & 428,4 & \\
\hline \multicolumn{3}{|c|}{ Hora acordar - Fim de semana } \\
\hline $12-23$ meses & 388,6 & \\
\hline 24-35 meses & 403,9 & $12,8(* *)$ \\
\hline 36 meses & 485,9 & \\
\hline
\end{tabular}

Abreviaturas: KW- teste de Kruskal-Wallis; ${ }^{* *} p<0,001,{ }^{* *} p<0,01$; Fonte: Dados de pesquisa 
acordavam mais tarde $(\mathrm{OM}=428,43)$, não existindo diferenças estatisticamente significativas (Tabela 2).

\section{Horas de sono nas sestas}

Concluiu-se que a maioria das crianças não dormia a sesta da manhã durante a semana, nem no fim de semana $(85,9 \%$ vs. $79,6 \%$ ), verificando-se diferenças estatisticamente significativas entre dormir a sesta da manhã durante a semana $(p=0,000)$ e ao fim de semana $(p=0,000)$, em função da idade da criança. Relativamente à sesta da manhã, quer durante a semana, quer no fim de semana, verificou-se a tendência de as crianças mais novas a realizarem, independentemente da sua duração, enquanto que as mais velhas (24-35 meses e 36 meses) não a realizavam (Tabela 3).

Apurou-se que a maioria das crianças realizava a sesta à tarde durante a semana $(94,7 \%)$ e no fim de semana $(93,1 \%)$, encontrando-se significância estatística apenas no fim de semana $(p=0,000)$. Verificou-se que as crianças mais novas (12-23 meses) tendem a dormir até uma hora de sesta e as crianças mais velhas (24-35 meses e 36 meses) não dormem a sesta (Tabela 3).

\section{Horas de sono nas $24 \mathrm{~h}$}

Concluiu-se que existem diferenças estatisticamente significativas em função da idade da criança relativamente ao número de horas que dormiam nas $24 \mathrm{~h}$, quer durante a semana $(p=0,000)$, quer ao fim de semana $(p=0,000)$.

Constatou-se que as crianças mais novas (12-23 meses) tendem a dormir mais tempo por dia (>14h) durante a semana. Ao fim de semana essa tendência mantém-se, observando-se que as crianças mais novas tendem a dormir mais tempo $(>14 \mathrm{~h}$ por dia) e as mais velhas, menos ( $<11 \mathrm{~h}$ ou 11-14h) (Tabela 4$)$.

Tabela 3. Duração da sesta da manhã e da tarde durante a semana e o fim de semana em função da idade da criança. Viseu, Portugal, 2019.

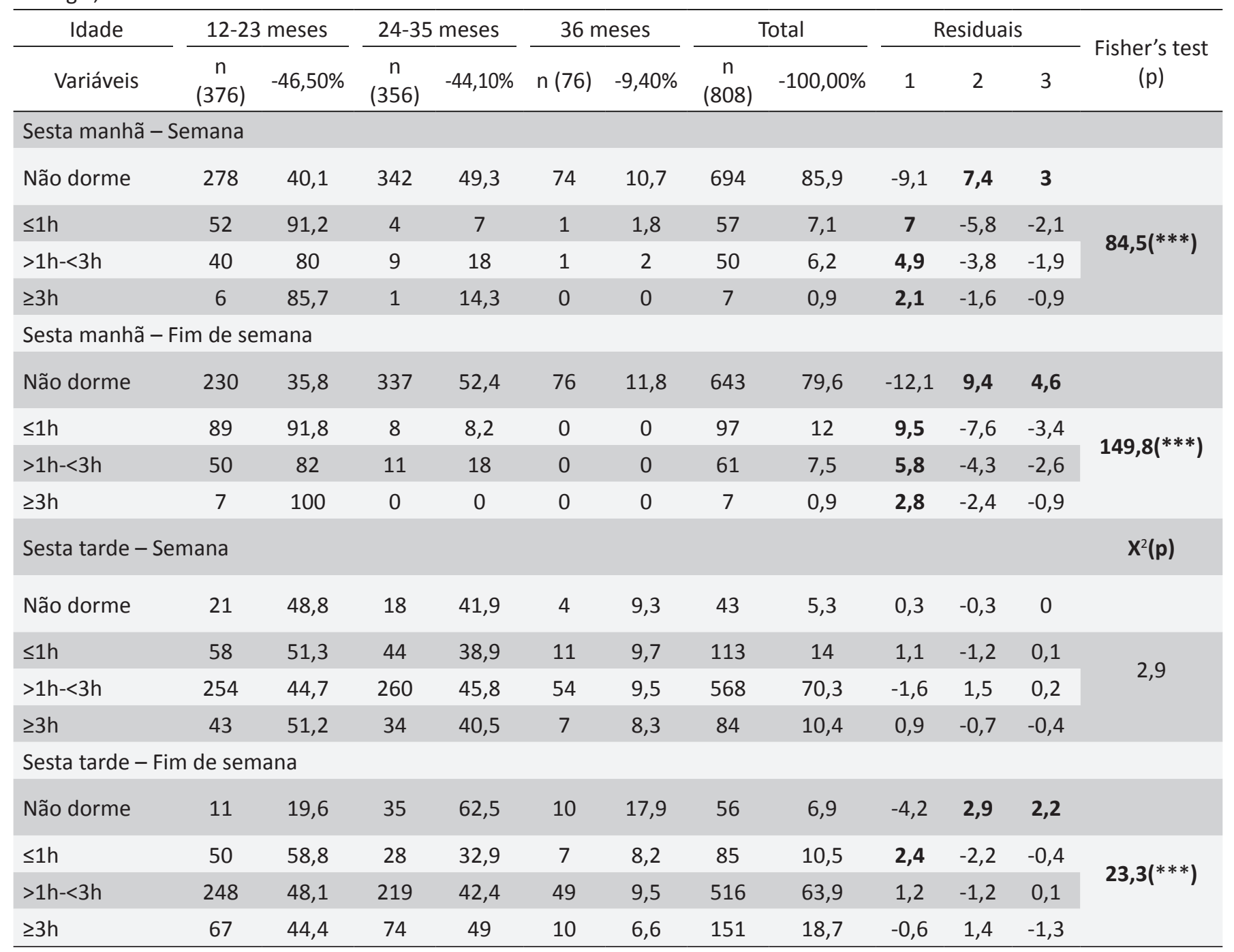

Abreviaturas: Fisher's test- Teste Exato de Fisher; $\mathrm{X}^{2}$ - teste de Qui quadrado; ${ }^{* * *} \mathrm{p}<0,001$; Fonte: Dados de pesquisa 
Tabela 4. Horas de sono nas $24 \mathrm{~h}$ durante a semana e o fim de semana em função da idade da criança. Viseu, Portugal, 2019.

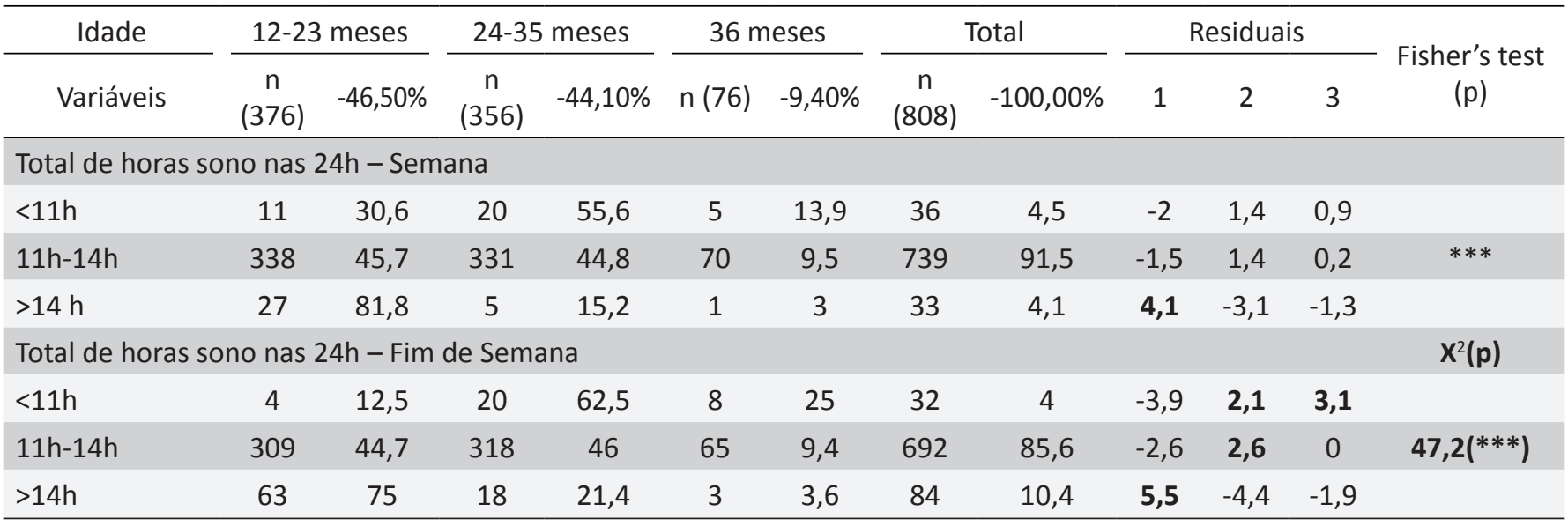

Abreviaturas: Fisher's test - Teste Exato de Fisher; $X^{2}$ - teste de Qui quadrado; ${ }^{* * *} p<0,001$; Fonte: Dados de pesquisa

Tabela 5. Estatística relativa ao total de horas de sono nas $24 \mathrm{~h}$ durante a semana e o fim de semana em função da idade da criança. Viseu, Portugal, 2019.

\begin{tabular}{|c|c|c|c|c|c|c|}
\hline Idade da criança & $\mathrm{n}$ & Min. & Máx. & $x$ & DP & CV\% \\
\hline \multicolumn{7}{|c|}{ Total de horas sono nas $24 \mathrm{~h}$ - Semana } \\
\hline $12-23$ meses & 376 & $10 \mathrm{~h}$ & $18 \mathrm{~h}$ & $12 \mathrm{~h} 40 \mathrm{~m}$ & $1 \mathrm{~h} 07 \mathrm{~m}$ & 8,63 \\
\hline 36 meses & 76 & $10 \mathrm{~h}$ & $14 \mathrm{~h} 30 \mathrm{~m}$ & $12 \mathrm{~h} 08 \mathrm{~m}$ & $52 m$ & 4,30 \\
\hline Total & 808 & $9 \mathrm{~h} 30 \mathrm{~m}$ & $18 \mathrm{~h}$ & $12 \mathrm{~h} 25 \mathrm{~m}$ & $1 \mathrm{~h} 02 \mathrm{~m}$ & 8,33 \\
\hline $12-23$ meses & 376 & $10 \mathrm{~h}$ & $19 \mathrm{~h}$ & $13 \mathrm{~h} 13 \mathrm{~m}$ & 1h $16 \mathrm{~m}$ & 8,83 \\
\hline 24-35 meses & 356 & $9 \mathrm{~h}$ & $17 \mathrm{~h}$ & $12 \mathrm{~h} 31 \mathrm{~m}$ & $1 \mathrm{~h} 08 \mathrm{~m}$ & 8,77 \\
\hline 36 meses & 76 & $9 h$ & $15 \mathrm{~h}$ & $12 \mathrm{~h} 18 \mathrm{~m}$ & 1h $09 \mathrm{~m}$ & 8,95 \\
\hline Total & 808 & $9 \mathrm{~h}$ & $19 \mathrm{~h}$ & $12 \mathrm{~h} 49 \mathrm{~m}$ & 1h $15 \mathrm{~m}$ & 9,21 \\
\hline
\end{tabular}

Fonte: Dados de pesquisa

O total de horas de sono por dia durante a semana, em função da idade das crianças, variou entre $9 \mathrm{~h} 30 \mathrm{~m}$ e $18 \mathrm{~h}(\mathrm{M}=12 \mathrm{~h} 25 \mathrm{~m}$ $\pm 1 \mathrm{~h} 02 \mathrm{~m})$. As crianças com idade entre os $12-23$ meses eram as que dormiam mais tempo por dia durante a semana $(M=12 \mathrm{~h} 40 \mathrm{~m}$ $\pm 1 \mathrm{~h} 07 \mathrm{~m})$. Durante o fim de semana, registrou-se uma variação entre $9 \mathrm{~h}$ e $19 \mathrm{~h}(\mathrm{M}=12 \mathrm{~h} 49 \mathrm{~m} \pm 1 \mathrm{~h} 15 \mathrm{~m})$; as crianças com idade entre 12-23 meses foram aquelas que dormiam mais $(M=13 \mathrm{~h}$ $13 \mathrm{~m} \pm 1 \mathrm{~h} 16 \mathrm{~m})$. Esse foi o grupo etário em que se registrou o valor mínimo e o máximo (10h e 19h) de horas totais de sono por dia (Tabela 5).

\section{DISCUSSÃO}

Neste estudo, efetuou-se a avaliação dos padrões de sono de crianças com idades entre 12 e 36 meses, considerando-se, durante a semana e no fim de semana, o sono noturno (hora de deitar e de acordar), o sono diurno (duração da sesta da manhã e da tarde) e as horas de sono totais (nas 24h). Os resultados foram apurados numa amostra de 808 crianças.

O sono é uma necessidade e a sua duração, regularidade e qualidade podem, muitas vezes, estar associadas aos hábitos familiares ${ }^{12}$. Os padrões de sono começam a definir-se melhor por volta dos cinco a seis meses de idade, quando ocorre a consolidação do sono. Os padrões de sono diurno tornam-se mais consistentes e consolidados mais tarde em comparação aos do sono noturno.

O sono saudável impõe duração e tempo adaptados à idade da criança e um horário regular para deitar a criança, durante a semana e o fim de semana, com uma diferença máxima de 30 minutos ${ }^{10}$. Neste estudo, verificou-se que o diferencial entre a média da hora de deitar as crianças durante a semana e no fim de semana era de 20 minutos ( $21 \mathrm{~h} 22 \mathrm{~m}$ à semana vs $21 \mathrm{~h} 42 \mathrm{~m}$ ao 
fim de semana). Essa variação pode indicar uma correta higiene do sono. Min Ahn e colaboradores ${ }^{13}$, no estudo que realizaram com crianças coreanas e em países/regiões predominantemente asiáticos ( $P-A)$ e predominantemente caucasianos $(P-C)$, do nascimento até aos 36 meses, revelaram, entre outros, resultados sobre os padrões de sono de crianças pequenas. Os autores verificaram que as crianças $\mathrm{P}-\mathrm{C}$, em média, se deitavam às $20 \mathrm{~h}$ $25 \mathrm{~m}$ e que igualmente se deitavam mais cedo ( $P-A=21 \mathrm{~h} 25 \mathrm{~m}$ e coreanas $=22 \mathrm{~h} 20 \mathrm{~m}$ ).

Concluiu-se que as crianças participantes deste estudo dormiam em média, por noite, $10 \mathrm{~h} 22 \mathrm{~m} \pm 42 \mathrm{~m}$ durante a semana e $10 \mathrm{~h} 36 \mathrm{~m} \pm 49 \mathrm{~m}$ ao fim de semana, o que se afigura compatível com as recomendações de entidades nacionais e internacionas ${ }^{10,11}$ de que o sono noturno tenha uma duração de 10 a 11 horas, verificando-se assim que as crianças tinham um padrão de sono adequado à idade.

Esses resultados são superiores aos apurados por outros autores, como Zhang e colaboradores ${ }^{5}$, que concluíram que, em média, a duração do sono noturno das 173 crianças, dos 12 aos 36 meses, que participaram do estudo, era de $10 \mathrm{~h} 09 \mathrm{~m}$, bem como os de Min Ahn e colaboradore ${ }^{13}$ que encontraram valores que oscilaram entre $9 \mathrm{~h} 12 \mathrm{~m}$ nas crianças $\mathrm{P}-\mathrm{A}, 9 \mathrm{~h} 42 \mathrm{~m}$ nas crianças coreanas e $10 \mathrm{~h} 01 \mathrm{~m}$ nas crianças P-C e, ainda, idênticos aos de Chindamo e colaboradore ${ }^{14}$ que, num estudo realizado em Itália, com 1.117 crianças de 12-36 meses, observaram uma média de $10 \mathrm{~h} 37 \mathrm{~m}$. Contudo, os resultados do presente estudo são inferiores aos de McDonald e colaboradores ${ }^{15}$, que verificaram a duração média do sono noturno de $11 \mathrm{~h} 06 \mathrm{~m}$ nas crianças entre 14 e 27 meses participantes do seu estudo.

A Sociedade Portuguesa de Pediatria (SPP) ${ }^{10}$ refere que, a partir dos 12 meses, a criança deve fazer três períodos de sono: um de noite (sono noturno) e dois de dia (sesta da manhã e da tarde). Entre os 15 e os 30 meses de idade, a criança, por norma, suspende de forma espontânea a sesta da manhã, preservando a da tarde, a qual só será abandonada entre os três e os cinco anos, ou mais tarde, dependendo da criança. Salienta-se que o sono diurno pode durar de $2 \mathrm{~h}$ a $4 \mathrm{~h}$ nas crianças mais jovens e $1 \mathrm{~h}$ a $3 \mathrm{~h}$ nas mais velhas. No presente estudo, verificou-se que a maioria das crianças já não realizava a sesta da manhã, mas mantinha a sesta da tarde, encontrando-se diferenças estatísticas significativas entre as crianças mais velhas e as do grupo que não dormia a sesta da manhã. A duração média das sestas, durante a semana, foi de $2 \mathrm{~h} 07 \mathrm{~m}$ e, no fim de semana, de $2 \mathrm{~h} 21 \mathrm{~m}$, dados que se revelaram consonantes aos apresentados pela SPP, e superiores aos encontrados noutros estudos ${ }^{15} \mathrm{em}$ que a média do sono diurno das crianças era de $1 \mathrm{~h} 09 \mathrm{~m}$.

No que concerne ao total de horas de sono diário, a literatura ${ }^{10,11}$ refere que, a partir dos 12 meses de idade, a duração diminui, variando entre $11 \mathrm{~h}$ e $14 \mathrm{~h}$. Na presente amostra verificou-se que a média de horas que as crianças dormiam, tanto durante a semana, quanto no fim de semana, se situava nesse intervalo (12h $25 \mathrm{~m}$ vs $12 \mathrm{~h} 49 \mathrm{~m}$, respetivamente); corroborando a evidência científica, eram as crianças mais velhas as que dormiam menos.
Esses resultados permitem-nos concluir que, em média, as crianças dormem o número de horas recomendado. Williamson e colaboradores ${ }^{16}$, num estudo que realizaram com uma amostra de 1983 crianças na faixa etária entre 18 e 36 meses, de vários países (Austrália, Canadá, Nova Zelândia, Reino Unido e Estados Unidos), concluíram, igualmente, que em todos esses países as taxas de sono diminuíam linearmente com a idade.

\section{CONSIDERAÇÕES FINAIS}

Este estudo permitiu avaliar os padrões de sono diurnos, noturnos e globais das crianças de 12 a 36 meses de idade durante a semana e no fim de semana. Assim, em concordância com o referencial teórico, que define um horário de sono diário para essa faixa etária entre $11 \mathrm{~h}$ e $14 \mathrm{~h}$, concluiu-se, no global da amostra, que em média as crianças dormiam o número de horas recomendado, embora uma pequena percentagem dormisse menos ou mais horas que o recomendado. Concluiu-se que a sesta da manhã não era realizada por grande parte das crianças da amostra estudada, independentemente da idade. Considera-se fundamental o reforço que os enfermeiros possam fazer sobre os benefícios da realização dessa sesta, quer para o desenvolvimento da criança, quer para a obtenção do número diário adequado de horas de sono.

Os resultados apurados confirmam, ainda, que, à medida que a idade avança, a necessidade de a criança dormir é menor, ou seja, a duração diária do sono reduz de forma progressiva a par do seu crescimento.

Promover uma boa higiene do sono nas crianças pequenas deve ser visto como um importante objetivo de saúde pública e enfermagem, pelas graves consequências que a sua ausência pode trazer ao nível do seu desenvolvimento. Os resultados desta investigação permitem concluir que é fundamental que os profissionais de saúde, nomeadamente os enfermeiros, continuem a direcionar a sua atenção para a definição de estratégias de intervenção específicas para os pais das crianças pequenas, tendo em vista a aquisição, a manutenção e o reforço da informação sobre o padrão de sono adequado, salientando os benefícios para a saúde e o desenvolvimento das crianças. Boas práticas de higiene do sono poderão promover um sono saudável nas crianças, influenciando os desfechos no nível da saúde e diminuindo a carga social dos tratamentos.

Importa referir que se encontraram algumas limitações neste estudo, nomeadamente: o fato de a informação coletada incidir unicamente sobre as horas de deitar, acordar e duração das sestas, não questionando hábitos e rotinas das crianças relativas ao sono; e também o fato de o instrumento de coleta de dados ser um questionário de autorresposta, o que poderá ser tido como uma limitação metodológica, uma vez que a veracidade dos resultados depende da sinceridade das respostas dos pais, isto é, as suas respostas podem ter sido mais orientadas para condutas que reconhecem como socialmente desejáveis, não traduzindo condutas reais, o que influencia os resultados do estudo. 


\section{CONTRIBUIÇÕES DOS AUTORES}

Desenho do estudo. Catarina Andreia Rosa Saraiva Marinho. Cândida Koch. Margarida Reis Santos.

Coleta de dados. Catarina Andreia Rosa Saraiva Marinho.

Análise de dados. Catarina Andreia Rosa Saraiva Marinho. João Carvalho Duarte.

Interpretação dos resultados. Catarina Andreia Rosa Saraiva

Marinho. Cândida Koch. Margarida Reis Santos. João Carvalho Duarte.

Redação e revisão crítica do manuscrito. Catarina Andreia Rosa Saraiva Marinho. João Carvalho Duarte. Cândida Koch. Margarida Reis Santos.

Aprovação da versão final do artigo. Catarina Andreia Rosa Saraiva Marinho. João Carvalho Duarte. Cândida Koch. Margarida Reis Santos.

Responsabilidade por todos os aspectos do conteúdo e a integridade do artigo publicado. Catarina Andreia Rosa Saraiva Marinho. João Carvalho Duarte. Cândida Koch. Margarida Reis Santos.

\section{EDITOR ASSOCIADO}

Aline Cristiane Cavachilli Okido (1)

\section{EDITOR CIENTÍFICO}

Ivone Evangelista Cabral (D)

\section{REFERÊNCIAS}

1. Bathory E, Tomopoulos S. Sleep regulation, physiology and development, sleep duration and patterns, and sleep hygiene in infants, toddlers, and preschool-age children. Curr Probl Pediatr Adolesc Health Care. 2017;47(2):29-42. http://dx.doi.org/10.1016/j.cppeds.2016.12.001. PMid:28117135.

2. Associação Portuguesa do Sono. Higiene do sono da criança e adolescente [Internet]. 2016 [citado 2021 jul 22]. Disponível em: http:// criancaefamilia.spp.pt/media/124389/HIGIENE SONO CRIANcA ADOLESCENTE.pdf

3. Karna B, Gupta V. Sleep disorder [Internet]. StatPearls Publishing; 2021 [citado 2021 jul 22]. Disponível em: https://www.ncbi.nlm.nih.gov/books/ NBK560720/

4. Rana M, Riffo Allende C, Mesa Latorre T, Rosso Astorga K, Torres AR. Sueño en los niňos: fisiología y actualizacion de los últimos conocimientos. Medicina. 2019;79(Supl. 3):25-8. PMid:31603839.
5. Zhang Z, Sousa-Sá E, Pereira J, Chaput J, Okely A, Feng X et al Correlates of nocturnal sleep duration, nocturnal sleep variability, and nocturnal sleep problems in toddlers: results from the get up! Study. Sleep Med. 2019;53:124-32. http://dx.doi.org/10.1016/j.sleep.2018.08.035. PMid:30508780.

6. de Bruin EJ, van Run C, Staaks J, Meijer AM. Effects of sleep manipulation on cognitive functioning of adolescents: a systematic review. Sleep Med Rev.2017 abr;32:45-57.http://dx.doi.org/10.1016/j.smrv.2016.02.006. PMid:27039223.

7. Paruthi S, Brooks LJ, D'Ambrosio C, Hall WA, Kotagal S, Lloyd RM et al. Consensus Statement of the American Academy of Sleep Medicine on the recommended amount of sleep for healthy children: methodology and discussion. J Clin Sleep Med. 2016;12(11):1549-61. http://dx.doi. org/10.5664/jcsm.6288. PMid:27707447.

8. Chaput J, Gray CE, Poitras VJ, Carson V, Gruber R, Birken CS et al. Systematic review of the relationships between sleep duration and health indicators in the early years (0-4 years). BMC Public Health 2017;17(Supl. 5):855. http://dx.doi.org/10.1186/s12889-017-4850-2. PMid:29219078.

9. National Sleep Foundation. How much sleep do we really need? [Internet] 2021 [citado 2021 jul 22]. Disponível em: https://www.sleepfoundation. org/how-sleep-works/how-much-sleep-do-we-really-need

10. Sociedade Portuguesa de Pediatria. Recomendações sps-spp: prática da sesta da criança nas creches e infantários, públicos ou privados [Internet]. 2017. p. 1-8 [citado 2021 jul 22]. Disponível em: http://www.metis. med.up.pt/index.php/Higiene_do_Sono_na_Infância_e_Adolescência

11. World Health Organization. Guidelines on physical activity, sedentary behaviour and sleep for children under 5 years of age [Internet]. Geneva: WHO; 2019 [citado 2021 jul 22]. Disponível em: https://apps.who.int/ iris/handle/10665/311664

12. Paruthi S, Brooks LJ, D'Ambrosio C, Hall WA, Kotagal S, Lloyd RM et al. Consensus statement of the American Academy of Sleep Medicine on the recommended amount of sleep for healthy children: methodology and discussion. J Clin Sleep Med. 2016;12(11):1549-61. http://dx.doi. org/10.5664/jcsm.6288. PMid:27707447.

13. Ahn Y, Williamson AA, Seo HJ, Sadeh A, Mindell JA. Sleep patterns among south korean infants and toddlers: global comparison. J Korean Med Sci. 2016;31(2):261-9. http://dx.doi.org/10.3346/jkms.2016.31.2.261. PMid:26839481.

14. Chindamo S, Buja A, DeBattisti E, Terraneo A, Marini E, Gomez Perez LJ et al. Sleep and new media usage in toddlers. Eur J Pediatr. 2019;178(4):483-90. http://dx.doi.org/10.1007/s00431-019-03318-7. PMid:30652219.

15. McDonald L, Wardle J, Llewellyn $\mathrm{CH}$, Van Jaarsveld C, Fisher A. Predictors of shorter sleep in early childhood. Sleep Med. 2014;15(5):536-40 http://dx.doi.org/10.1016/j.sleep.2014.01.005. PMid:24726571.

16. Williamson AA, Leichman ES, Walters RM, Mindell JA. Caregiverperceived sleep outcomes in toddlers sleeping in cribs versus beds. Sleep Med. 2019;54:16-21. http://dx.doi.org/10.1016/.sleep.2018.10.012. PMid:30529772. 